SCALING LIMITS OF THE CHERN-SIMONS-HIGGS ENERGY

By

Matthias Kurzke

and

Daniel Spirn

IMA Preprint Series \# 2130

(August 2006)

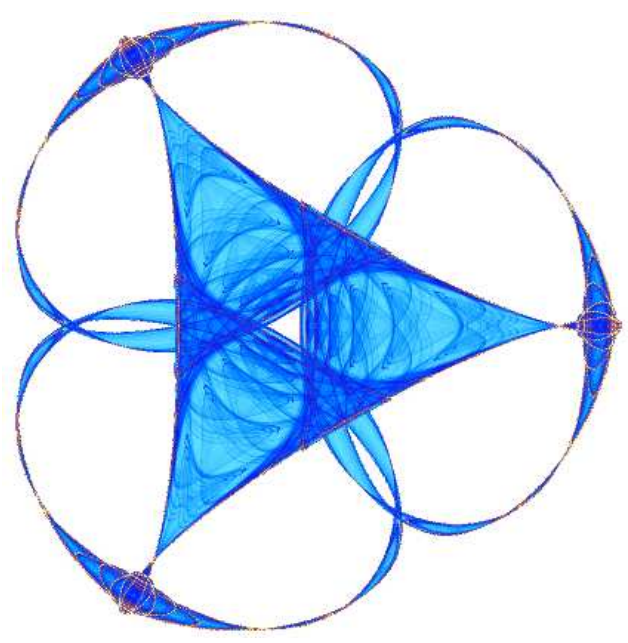

INSTITUTE FOR MATHEMATICS AND ITS APPLICATIONS

UNIVERSITY OF MINNESOTA 400 Lind Hall

207 Church Street S.E.

Minneapolis, Minnesota 55455-0436

Phone: 612-624-6066 Fax: 612-626-7370

URL: http://www.ima.umn.edu 


\title{
SCALING LIMITS OF THE CHERN-SIMONS-HIGGS ENERGY
}

\author{
MATTHIAS KURZKE AND DANIEL SPIRN
}

Abstract. We continue our study in [16] of the Gamma limit of the Abelian Chern-Simons-Higgs energy

$$
G_{c s h}:=\frac{1}{2} \int_{U}\left|\nabla_{A_{\varepsilon}} u_{\varepsilon}\right|^{2}+\frac{\mu_{\varepsilon}^{2}}{4} \frac{\left|\operatorname{curl} A_{\varepsilon}-h_{e x}\right|^{2}}{\left|u_{\varepsilon}\right|^{2}}+\frac{1}{\varepsilon^{2}}\left|u_{\varepsilon}\right|^{2}\left(1-\left|u_{\varepsilon}\right|^{2}\right)^{2} d x
$$

on a bounded, simply connected, two dimensional domain where $\varepsilon \rightarrow 0$ and $\mu_{\varepsilon} \rightarrow \mu \in[0,+\infty]$. Under the critical scaling, $G_{c s h} \approx|\log \varepsilon|^{2}$, we establish the Gamma limit when $\mu \in(0,+\infty]$, and as a consequence we are able to compute the first critical field $H_{1}=H_{1}(U, \mu)$ for the nucleation of a vortex. Finally, we show failure of Gamma convergence when $\mu_{\varepsilon} \rightarrow 0$ (this includes the self-dual case). The method entails estimating in certain weak topologies the Jacobian $J\left(u_{\varepsilon}\right)=\operatorname{det}\left(\nabla u_{\varepsilon}\right)$ in terms of the Chern-Simons-Higgs energy $E_{c s h}$.

\section{INTRODUCTION}

Abelian Chern-Simons-Higgs (CSH) theory serves as an anyon model $[4,9$, $8,24]$ and is a classical field theory defined on $(2+1)$ dimensional Minkowski space. Such models have applications to the theory of high temperature superconductivity, quantum Hall effects and carry fractional charge values [4, 24]. This model has been the source of much interest in the physics community; the book of Yang [24] offers an excellent overview of Chern-Simons-Higgs and related theories. Letting $D_{\alpha}=\left(-\partial_{\Phi}, \nabla_{A}\right)$ then the CSH Lagrangian has the form

$$
\mathcal{L}_{C S H}=\int D_{\alpha} \overline{D^{\alpha} u}+\frac{\mu^{2}}{4} \epsilon^{\alpha \beta \gamma} A_{\alpha} F_{\beta \gamma}-\frac{1}{\varepsilon^{2}}|u|^{2}\left(1-|u|^{2}\right)^{2}
$$

where $\epsilon^{\alpha \beta \gamma}$ is the antisymmetric tensor. Here $F_{\alpha}$ is the Maxwell curvature tensor and $\epsilon^{\alpha \beta \gamma} A_{\alpha} F_{\beta \gamma}$ is the Chern-Simons term. The associated Euler-Lagrange

D. Spirn was supported in part by NSF grant DMS-0510121. 
(CSH) equations are:

$$
\begin{aligned}
\partial_{\Phi}^{2} u & =\nabla_{A}^{2} u+\frac{1}{\varepsilon^{2}} u\left(1-|u|^{2}\right)\left(3|u|^{2}-1\right) \\
q & =-\frac{\mu^{2}}{2} \operatorname{curl} A \\
j_{A} & =\frac{\mu^{2}}{2}\left(E \times e_{3}\right)
\end{aligned}
$$

where $q=\left(i u, \partial_{\Phi} u\right), j_{A}=\left(i u, \nabla_{A} u\right), E=\partial_{t} A-\nabla \Phi$, and $h=\operatorname{curl} A$.

Since $u: \mathbb{R}^{2} \rightarrow \mathbb{C}$ we can induce the formation of topological vortices regions where $|u|=0$ and about which the winding number of the phase is nontrivial. Setting $u=\rho e^{i \varphi} \approx e^{i \varphi}$ over $\mathbb{R}^{2}$ and $\varphi=d \theta$ with $d \in \mathbb{Z}$, then $J_{A} \approx \operatorname{curl}(\nabla \varphi-A)=\operatorname{curl} \nabla \varphi-h$. Formally, if we integrate the curl of (3) over $\mathbb{R}^{2}$ then $2 \pi d=\int_{\mathbb{R}^{2}} h d x$. Furthermore, integrating (2) over the plane yields

$$
d=\frac{1}{2 \pi} \int_{\mathbb{R}^{2}} h d x=-\frac{1}{\mu^{2} \pi} \int_{\mathbb{R}^{2}} q d x .
$$

As in Ginzburg-Landau theory, we see that the current and the magnetic field are quantized about a topological vortex; however, in CSH theory the magnetic field to induces a quantized electric charge, which can have arbitrary values, depending on $\mu$. This quantized electric charge is a fundamental feature of Chern-Simons-Higgs theory.

Since the CSH equations serve as a model for high temperature superconductors, we include the possible presence of an applied magnetic field $h_{e x}$. If we look for solutions of (1)-(3) that are independent of time by setting $\partial_{t} u \equiv 0$, then we can remove the electric field potential $\Phi$, and we are left with a set of coupled elliptic PDE's:

$$
\begin{aligned}
-\frac{\mu^{2}}{4} \frac{\left|\operatorname{curl} A-h_{e x}\right|^{2}}{|u|^{4}} u & =\nabla_{A}^{2} u+\frac{1}{\varepsilon^{2}} u\left(1-|u|^{2}\right)\left(3|u|^{2}-1\right) \\
0 & =-\frac{\mu^{2}}{4} \operatorname{curl}\left(\frac{\operatorname{curl} A-h_{e x}}{|u|^{2}}\right)+j_{A}(u) .
\end{aligned}
$$

Equations (4)-(5) can be viewed as the Euler-Lagrange equations of the following Chern-Simons-Higgs energy

$$
G_{c s h}\left(u, A ; h_{e x}\right)=\frac{1}{2} \int_{U}\left|\nabla_{A} u\right|^{2}+\frac{\mu^{2}}{4} \frac{\left|\operatorname{curl} A-h_{e x}\right|^{2}}{|u|^{2}}+\frac{1}{\varepsilon^{2}}|u|^{2}\left(1-|u|^{2}\right)^{2} d x
$$

for an applied magnetic field, $h_{e x}$, and a bounded, simply connected domain, $U \subset \mathbb{R}^{2}$. A discussion of the CSH theory on bounded domains can be found in $[6]$. 
We briefly describe some features of CSH topological vortices. First of all magnetic fields concentrate in an annular region about each topological vortex. This is in contrast to Ginzburg-Landau vortices, where the magnetic field concentrates at the site of the vortex. This concentration behavior is due to the $\frac{1}{|u|^{2}}$ part of the second term of (6). Second, in the $\varepsilon \rightarrow 0$ limit $\left|u_{\varepsilon}\right|$ relaxes to $\mathbb{S}^{1} \cup\{0\}$, as opposed to $\mathbb{S}^{1}$ in the Ginzburg-Landau case. This implies that nontopological vortices (regions where $|u|=0$ with trivial winding number about the region) are possible and potentially favorable. However, that such regions are of size $O(\varepsilon)$ if $\left|u_{\varepsilon}\right| \geq c_{0}>0$ on $\partial U$ for some constant $c_{0}=c_{0}(U)$, see [16].

1.1. Prior results. Up to now, most attention has focussed on the self-dual case where $\mu_{\varepsilon} \equiv \varepsilon$ and $h_{e x} \equiv 0$. In this case the CSH equations reduce, following Hong-Kim-Pac and Jackiw-Weinberg [8, 9], to a system of first order PDE's. Solutions can be recovered by solving (after a substitution) a Liouvilletype elliptic equation, similar to the Jaffe-Taubes approach to solving self-dual solutions in Ginzburg-Landau theory [10]. It is impossible to give an adequate accounting of the extensive results on self-dual solutions to the Chern-SimonsHiggs equations, but we direct the reader to $[4,5,6,8,9,17,23,24]$ and the references therein.

We turn our attention to non self-dual Chern-Simons-Higgs theory. The first rigorous results to our knowledge for small $\varepsilon$ and $\mu=O(1)$ for the CSH functional are those of Han-Kim [7], who studied sequential minimizers $\left\{u_{\varepsilon}, A_{\varepsilon}\right\}$ of (6) with $A_{\varepsilon} \equiv 0$ and Dirichlet boundary condition $u_{\varepsilon}=g$ on $\partial U$ with $|g|=1$. Their proofs are similar in spirit to Bethuel-Brezis-Helein [2] for the simplified Ginzburg-Landau energy

$$
E_{g l}(u)=\frac{1}{2} \int_{U}|\nabla u|^{2}+\frac{1}{2 \varepsilon^{2}}\left(1-|u|^{2}\right)^{2}
$$

and rely heavily on the maximum principle for $\left|u_{\varepsilon}\right|$. The maximum principle fails when gauge field $A_{\varepsilon} \not \equiv 0$, so another approach is needed.

In [16] the authors studied (6) with $A_{\varepsilon} \not \equiv 0$ in the $\Gamma$-convergence framework under various energy scalings for $\varepsilon \rightarrow 0$ and $\mu_{\varepsilon} \equiv \mu \in(0,+\infty)$. The techniques used are related to the approach of Jerrard-Soner $[12,13]$ combined with the Sandier [18] version of the vortex ball construction method of Jerrard [11] and Sandier [18]. Similar to their approach to studying (7), we first study the simplified functional

$$
E_{c s h}(u)=\frac{1}{2} \int_{U}|\nabla u|^{2}+\frac{1}{\varepsilon^{2}}|u|^{2}\left(1-|u|^{2}\right)^{2}
$$


which helps us express the energetic limit of $G_{c s h}$. Consider $v_{\varepsilon}=\frac{j\left(u_{\varepsilon}\right)}{r_{\varepsilon}}, a_{\varepsilon}=\frac{A_{\varepsilon}}{s_{\varepsilon}}$ and $h_{e x}=\frac{H}{t_{\varepsilon}}$ then

$$
\begin{aligned}
G_{c s h}\left(u_{\varepsilon}, A_{\varepsilon} ; h_{e x}\right) \approx E_{c s h}\left(\left|u_{\varepsilon}\right|\right)+\frac{r_{\varepsilon}^{2}}{2}\left[\left\|v_{\varepsilon}-\frac{s_{\varepsilon}}{r_{\varepsilon}} a_{\varepsilon}\right\|_{L^{2}\left(U \backslash \cup B_{r_{j}}\right)}^{2}\right. \\
\left.+\frac{|\log \varepsilon|}{r_{\varepsilon}}\left\|\operatorname{curl} v_{\varepsilon}\right\|_{\mathcal{M}\left(\cup B_{r_{j}}\right)}+\frac{\mu_{\varepsilon}^{2}}{4}\left\|\frac{\frac{s_{\varepsilon}}{r_{\varepsilon}} \operatorname{curl} a_{\varepsilon}-\frac{t_{\varepsilon}}{r_{\varepsilon}} H}{\left|u_{\varepsilon}\right|}\right\|_{L^{2}(U)}^{2}\right] .
\end{aligned}
$$

Such an energetic framework was proved in [16]. The critical scaling $G_{c s h} \approx$ $|\log \varepsilon|^{2}$ is the energy scaling where the order parameter interacts at the same order as the induced magnetic field, and we can see this from a simple scaling argument. Formally, for the induced magnetic field to interact with the order parameter we need both the $\operatorname{curl} v_{\varepsilon}$ and $v_{\varepsilon}$ terms, so

$$
r_{\varepsilon} \equiv|\log \varepsilon| \quad s_{\varepsilon} \equiv r_{\varepsilon}
$$

and for the external magnetic field to interact with the induced magnetic field we need

$$
t_{\varepsilon} \equiv r_{\varepsilon}
$$

regardless of $\mu_{\varepsilon}$. Thus,

$$
G_{c s h}\left(u_{\varepsilon}, A_{\varepsilon} ; h_{e x}\right) \approx \frac{|\log \varepsilon|^{2}}{2}\left[\left\|v_{\varepsilon}-a_{\varepsilon}\right\|_{L^{2}}^{2}+\left\|\operatorname{curl} v_{\varepsilon}\right\|_{\mathcal{M}}+\frac{\mu_{\varepsilon}^{2}}{4}\left\|\operatorname{curl} a_{\varepsilon}-H\right\|_{L^{2}}^{2}\right] .
$$

Therefore, we can describe the $G_{c s h} \approx|\log \varepsilon|^{2}$ energy scaling as "critical".

In [16] we study $G_{c s h} \approx|\log \varepsilon|^{2}$ for $\mu_{\varepsilon} \equiv \mu \in(0,+\infty)$, among other asymptotic limits. Our general assumptions are that $U \subset \mathbb{R}^{2}$ is a bounded, simply connected domain with smooth boundary. We take $\left\|u_{\varepsilon}\right\|_{L^{\infty}(U)} \leq C<+\infty$, so there are no nontopological vortices. For simplicity, we state the result in the Coulomb gauge, which amounts to considering only pairs $(u, A)$ with $\nabla \cdot A=0$ in $U$ and $A \cdot \nu=0$ on $\partial U$. These conditions can always be satisfied by an appropriate gauge transformation replacing $(u, A)$ by $\left(u e^{i \chi}, A+\nabla \chi\right)$ without changing the energy. Finally, we assume that $\left\{u_{\varepsilon}\right\}$ is a sequence of functions in $H^{1}(U ; \mathbb{C})$ whose traces on $\partial U$ satisfy $\left|u_{\varepsilon}\right| \geq 1-\frac{1}{|\log \varepsilon|}$, although we believe this assumption is technical and not crucial to the results. We recall the following results, stated here in the spirit of $\Gamma$-convergence; that is, separated into a compactness result combined with a lower bound for the energy and a construction that shows that the lower bound is essentially optimal.

Theorem 1 ([16]). Assume that the external field satisfies $h_{e x}=H|\log \varepsilon|$ for some $H>0$ and $\mu_{\varepsilon} \rightarrow \mu \in(0,+\infty)$. Consider a sequence $\left\{u_{\varepsilon}, A_{\varepsilon}\right\}$ with

$$
G_{c s h}\left(u_{\varepsilon}, A_{\varepsilon} ; h_{e x}\right) \leq K|\log \varepsilon|^{2},
$$


$\left|u_{\varepsilon}\right| \geq 1-\frac{1}{|\log \varepsilon|}$ on $\partial U$, and $\left\|u_{\varepsilon}\right\|_{L^{\infty}(U)} \leq C$. Set $a_{\varepsilon}=\frac{1}{|\log \varepsilon|} A_{\varepsilon}, v_{\varepsilon}=\frac{1}{|\log \varepsilon|} j\left(u_{\varepsilon}\right)$, and $w_{\varepsilon}=\frac{J\left(u_{\varepsilon}\right)}{|\log \varepsilon|}$. Then $\left\{a_{\varepsilon}\right\}$ is weakly precompact in $H^{1}, v_{\varepsilon}=\frac{1}{|\log \varepsilon|} j\left(u_{\varepsilon}\right)$ converges to $v$ weakly in all $L^{p}, \frac{v_{\varepsilon}}{\left|u_{\varepsilon}\right|} \rightarrow v$ in $L^{2}$, and $w_{\varepsilon}=\frac{J\left(u_{\varepsilon}\right)}{|\log \varepsilon|} \rightarrow w=\frac{1}{2} \operatorname{curl} v$ in Radon measure. Furthermore, the energy satisfies

$$
\begin{aligned}
\liminf _{\varepsilon \rightarrow 0} & \frac{1}{|\log \varepsilon|^{2}} G_{c s h}\left(u_{\varepsilon}, A_{\varepsilon} ; h_{e x}\right) \\
& \geq G(v, a ; H)=\frac{1}{2}\left(\int_{U}|v-a|^{2}+\frac{\mu^{2}}{4}|\operatorname{curl} a-H|^{2}+\|\operatorname{curl} v\|_{\mathcal{M}}\right) .
\end{aligned}
$$

Conversely, for any $a \in H^{1}\left(U ; \mathbb{R}^{2}\right)$ and $v \in L^{2}\left(U ; \mathbb{R}^{2}\right)$ such that $w=\frac{1}{2} \operatorname{curl} v$ is a Radon measure, there exists a sequence $\left\{u_{\varepsilon}\right\}$ in $H^{1}(U ; \mathbb{C})$ with $\left|u_{\varepsilon}\right|=1$ on $\partial U$ and a sequence $\left\{A_{\varepsilon}\right\} \in H^{1}(U ; \mathbb{C})$ in Coulomb gauge such that $v_{\varepsilon}=\frac{1}{|\log \varepsilon|} j\left(u_{\varepsilon}\right) \rightarrow$ $v$ in $L^{2}, w_{\varepsilon}=\frac{1}{|\log \varepsilon|} J\left(u_{\varepsilon}\right) \rightarrow w$ in $\left(C^{0, \beta}\right)^{*}, a_{\varepsilon}=\frac{1}{|\log \varepsilon|} A_{\varepsilon} \rightarrow a$ in $H^{1}$, and such that (9) holds with equality.

Although Theorem 1 was established with $\mu_{\varepsilon} \equiv \mu \in(0,+\infty)$ a fixed constant, the $\mu_{\varepsilon} \rightarrow \mu \in(0,+\infty)$ case is a straightforward adaptation of the argument in Section 7 of [16]. As an application of the last theorem, we calculate the critical field $h_{c r i t}$ for which vortices appear in nonzero minimizers of $G_{c s h}\left(u_{\varepsilon}, A_{\varepsilon} ; h_{e x}\right)$.

Corollary 2 ([16]). As $\varepsilon \rightarrow 0$, the critical field $h_{\text {crit }}$ is given asymptotically by $H_{1}(\mu)|\log \varepsilon|$, where

$$
H_{1}(\mu)=\frac{2}{\mu^{2} \max _{\bar{U}}\left|z_{\mu}\right|}
$$

and $z_{\mu}$ is the solution of

$$
-\frac{\mu^{2}}{4} \Delta z_{\mu}+z_{\mu}+1=0 \text { in } U \quad z_{\mu}=0 \text { on } \partial U .
$$

Concerning the dependence on $\mu$, we have that $\mu^{2} H_{1}(\mu) \rightarrow 2$ as $\mu \rightarrow 0$. Furthermore, $H_{1}(\mu)$ is decreasing in $\mu$ and converges to a limit $\bar{H}(U)>0$ as $\mu \rightarrow \infty$.

The corollary follows from Theorem 1 using some analysis of the limit functional.

1.2. Results. It is natural to ask whether the formal behavior of $H_{1}$ as $\mu \rightarrow 0$ or $\mu \rightarrow \infty$ in Corollary 2 hold in a rigorous sense. In particular we let $\mu_{\varepsilon} \rightarrow$ $\mu \in\{0,+\infty\}$ and consider the $\Gamma$-limit of

$$
G_{c s h}\left(u, A ; h_{e x}\right)=\frac{1}{2} \int_{U}\left|\nabla_{A_{\varepsilon}} u_{\varepsilon}\right|^{2}+\frac{\mu_{\varepsilon}^{2}}{4} \frac{\left|\operatorname{curl} A_{\varepsilon}-h_{e x}\right|^{2}}{\left|u_{\varepsilon}\right|^{2}}+\frac{1}{\varepsilon^{2}}\left|u_{\varepsilon}\right|^{2}\left(1-\left|u_{\varepsilon}\right|^{2}\right)^{2}
$$

at the critical energy scaling $G_{c s h} \approx|\log \varepsilon|^{2}$. In the $\mu_{\varepsilon} \rightarrow+\infty$ we have 
Theorem 3 (Large $\mu_{\varepsilon}$ limit). Assume $\mu_{\varepsilon} \rightarrow \infty$ and $G_{c s h}\left(u_{\varepsilon}, A_{\varepsilon} ; h_{e x}\right) \leq$ $K|\log \varepsilon|^{2}$. Furthermore, assume $\left\|u_{\varepsilon}\right\|_{L^{\infty}(U)} \leq C<+\infty$ and $\left\|u_{\varepsilon}\right\|_{L^{\infty}(\partial U)} \geq$ $1-\frac{1}{|\log \varepsilon|}$. Assume that each $\left(u_{\varepsilon}, A_{\varepsilon}\right)$ is in Coulomb gauge, that is, $\nabla \cdot A_{\varepsilon}=0$ in $U$ and $A_{\varepsilon} \cdot \nu=0$ on $\partial U$.

Set $h_{e x}=H|\log \varepsilon|, a_{\varepsilon}=\frac{1}{|\log \varepsilon|} A_{\varepsilon}$, and $v_{\varepsilon}=\frac{1}{|\log \varepsilon|} j\left(u_{\varepsilon}\right)$, then the following compactness and convergence statements hold:

(1) $a_{\varepsilon} \rightarrow a=H a_{1}$ strongly in $H^{1}$, where $a_{1}$ is the solution of the system

$$
\begin{aligned}
& \operatorname{curl} a_{1}=0 \quad \text { in } U \\
& \nabla \cdot a_{1}=0 \quad \text { in } U \\
& a_{1} \cdot \nu=0 \text { on } \partial U \text {. }
\end{aligned}
$$

(2) For a subsequence, $v_{\varepsilon} \rightarrow v$ in all $L^{p}$ with $p<2$ and $\frac{v_{\varepsilon}}{\left|u_{\varepsilon}\right|} \rightarrow v$ in $L^{2}$. Furthermore, $\frac{J\left(u_{\varepsilon}\right)}{|\log \varepsilon|} \rightarrow \frac{1}{2} \operatorname{curl} v$ in $\left(C^{0, \beta}\right)^{*}$.

The energy satisfies the $\Gamma$-lim inf inequality

$$
\liminf _{\varepsilon \rightarrow 0} \frac{1}{|\log \varepsilon|^{2}} G_{c s h}\left(u_{e}, A_{\varepsilon} ; h_{e x}\right) \geq G(v)=\frac{1}{2}\left(\int_{U}|v-a|^{2}+\|\operatorname{curl} v\|_{\mathscr{M}}\right) .
$$

A gauge-invariant form can be given as

$$
G(v, a ; H)= \begin{cases}G(v) & \text { if } \operatorname{curl} a=H \\ +\infty & \text { else. }\end{cases}
$$

Conversely, for any $a \in H^{1}\left(U ; \mathbb{R}^{2}\right)$ and $v \in L^{2}\left(U ; \mathbb{R}^{2}\right)$ such that $w=\frac{1}{2} \operatorname{curl} v$ is a Radon measure, there exists a sequence $\left\{u_{\varepsilon}\right\}$ in $H^{1}(U ; \mathbb{C})$ with $\left|u_{\varepsilon}\right|=1$ on $\partial U$ and a sequence $\left\{A_{\varepsilon}\right\} \in H^{1}(U ; \mathbb{C})$ in Coulomb gauge such that $v_{\varepsilon}=$ $\frac{1}{|\log \varepsilon|} j\left(u_{\varepsilon}\right) \rightarrow v$ in $L^{2}, w_{\varepsilon}=\frac{1}{|\log \varepsilon|} J\left(u_{\varepsilon}\right) \rightarrow w$ in $\left(C^{0, \beta}\right)^{*}, a_{\varepsilon}=\frac{1}{|\log \varepsilon|} A_{\varepsilon} \rightarrow$ a in $H^{1}$, and such that (10) holds with equality.

The proof of Theorem 3 relies on a $\Gamma$-convergence result for $E_{c s h}\left(u_{\varepsilon}\right)$ established in [16], see the Appendix.

As an application of the last theorem, we calculate the critical field $h_{\text {crit }}$ for which vortices appear in nonzero minimizers of $G_{c s h}\left(u_{\varepsilon}, A_{\varepsilon} ; h_{e x}\right)$.

Corollary 4 (Large $\mu_{\varepsilon}$ critical field). As $\varepsilon \rightarrow 0$ and $\mu_{\varepsilon} \rightarrow+\infty$, the critical field $h_{\text {crit }}$ is given asymptotically by $H_{1}|\log \varepsilon|$, where

$$
H_{1}=\frac{2}{\max _{\bar{U}}\left|z_{\infty}\right|}
$$

and $z_{\infty}$ is the solution of

$$
-\Delta z_{\infty}+1=0 \text { in } U \quad z_{\infty}=0 \text { on } \partial U .
$$

Hence the $\mu \rightarrow \infty$ behavior of $H_{1}$ in Corollary 2 holds rigorously. . 
For the $\mu_{\varepsilon} \rightarrow 0$ case we find a failure of $\Gamma$-convergence in the critical $G_{c s h} \approx$ $|\log \varepsilon|^{2}$ scaling. In particular there can be a concentration phenomena of vortices at a single point, since a topological vortex costs very little energy when $\mu_{\varepsilon} \ll 1$. Therefore, we can pack vortices very close together and still make $G_{c s h} \approx|\log \varepsilon|^{2}$.

Theorem 5 (Small $\mu_{\varepsilon}$ compactness failure). For any $\mu_{\varepsilon} \rightarrow 0$, there exists a sequence $\left\{u_{\varepsilon}, A_{\varepsilon}\right\}$ with $G_{c s h}\left(u_{\varepsilon}, A_{\varepsilon} ; 0\right) \leq K|\log \varepsilon|^{2}$ such that $\frac{1}{|\log \varepsilon|} j\left(u_{\varepsilon}\right)$ and $\frac{1}{|\log \varepsilon|} \frac{j\left(u_{\varepsilon}\right)}{\left|u_{\varepsilon}\right|}$ are not bounded in $L^{2}$.

Remark 6. In particular Theorem 5 includes the self-dual case $\mu_{\varepsilon}=\varepsilon$ and $h_{e x}=0$; therefore, the $\Gamma$-limit fails for large numbers of vortices.

\section{LARGE $\mu_{\varepsilon}$}

The case of very large $\mu_{\varepsilon}$ turns out to be very similar to that of finite $\mu_{\varepsilon}$, and we obtain a restricted limit functional by a similar $\Gamma$-convergence argument.

Proof of Theorem 3. After rescaling, the CSH energy satisfies

$G_{c s h}\left(u_{\varepsilon}, A_{\varepsilon} ; h_{e x}\right)=E_{c s h}\left(\left|u_{\varepsilon}\right|\right)+\frac{|\log \varepsilon|^{2}}{2} \int_{U}\left|\frac{v_{\varepsilon}}{\left|u_{\varepsilon}\right|}-a_{\varepsilon}\right| u_{\varepsilon}||^{2}+\frac{\mu_{\varepsilon}^{2}}{4} \frac{\left|\operatorname{curl} a_{\varepsilon}-H\right|^{2}}{\left|u_{\varepsilon}\right|^{2}} d x ;$

therefore,

$$
\begin{aligned}
\frac{\mu_{\varepsilon}^{2}}{8} \int_{U}\left|\operatorname{curl} a_{\varepsilon}-H\right|^{2} d x & =\frac{\mu_{\varepsilon}^{2}}{8} \int_{U} \frac{\left|\operatorname{curl} a_{\varepsilon}-H\right|^{2}\left|u_{\varepsilon}\right|^{2}}{\left|u_{\varepsilon}\right|^{2}} d x \\
& \leq \frac{C^{2} \mu_{\varepsilon}^{2}}{8} \int_{U} \frac{\left|\operatorname{curl} a_{\varepsilon}-H\right|^{2}}{\left|u_{\varepsilon}\right|^{2}} d x \\
& \leq C^{2} \frac{G_{c s h}\left(u_{\varepsilon}, A_{\varepsilon} ; h_{e x}\right)}{|\log \varepsilon|^{2}} \\
& \leq C^{2} K
\end{aligned}
$$

where $C \geq\left\|u_{\varepsilon}\right\|_{L^{\infty}(U)}$ is one of our assumptions. From $\int_{U}\left|\operatorname{curl} a_{\varepsilon}-H\right|^{2} \leq$ $\frac{8 C^{2} K}{\mu_{\varepsilon}^{2}} \rightarrow 0$ as $\varepsilon \rightarrow 0$ we obtain that $\operatorname{curl} a_{\varepsilon} \rightarrow H$ strongly in $L^{2}, \operatorname{so} \operatorname{curl}\left(a_{\varepsilon}-\right.$ $\left.H a_{1}\right) \rightarrow 0$ in $L^{2}$. This, combined with the Coulomb gauge condition, implies elliptic regularity, and in turn the strong convergence of $\left(a_{\varepsilon}-H a_{1}\right) \rightarrow 0$ in $H^{1}(U)$.

The compactness assertions for $v_{\varepsilon}$ follow as in [16]: We decompose

$$
\begin{aligned}
G_{c s h}\left(u_{\varepsilon}, A_{\varepsilon} ; h_{e x}\right)= & E_{c s h}\left(u_{\varepsilon}\right)-\int_{U} j\left(u_{\varepsilon}\right) \cdot A_{\varepsilon} \\
& +\int_{U} \frac{1}{2}\left|A_{\varepsilon}\right|^{2}\left|u_{\varepsilon}\right|^{2}+\frac{\mu_{\varepsilon}^{2}}{8} \frac{\left|\operatorname{curl} A_{\varepsilon}-h_{e x}\right|^{2}}{\left|u_{\varepsilon}\right|^{2}}
\end{aligned}
$$


from which we see $E_{c s h}\left(u_{\varepsilon}\right) \leq G_{c s h}\left(u_{\varepsilon}, A_{\varepsilon} ; h_{e x}\right)+\int_{U} j\left(u_{\varepsilon}\right) \cdot A_{e}$, and we can repeat our reasoning of [16] (which in turn is adapted from [13]) and estimate

$$
\begin{aligned}
\left|A_{\varepsilon} \cdot j\left(u_{\varepsilon}\right)\right| & \leq \frac{1}{4} \frac{\left|j\left(u_{\varepsilon}\right)\right|^{2}}{\left|u_{\varepsilon}\right|^{2}}+\left|u_{\varepsilon}\right|^{2}\left|A_{\varepsilon}\right|^{2} \\
& \leq \frac{1}{4}\left|\nabla u_{\varepsilon}\right|^{2}+\left(\left.\left|u_{\varepsilon}\right||1-| u_{\varepsilon}\right|^{2} \mid+1\right)\left|A_{\varepsilon}\right|^{2} \\
& \leq \frac{1}{4}\left|\nabla u_{\varepsilon}\right|^{2}+\left|A_{\varepsilon}\right|^{2}+\frac{1}{4 \varepsilon^{2}}\left|u_{\varepsilon}\right|^{2}\left(1-\left|u_{\varepsilon}\right|^{2}\right)^{2}+2 \varepsilon^{2}\left|A_{\varepsilon}\right|^{4} \\
& \leq \frac{1}{2} E_{c s h}\left(u_{\varepsilon}\right)+\left|A_{\varepsilon}\right|^{2}+2 \varepsilon^{2}\left|A_{\varepsilon}\right|^{4},
\end{aligned}
$$

where we used the simple estimate $x^{2} \leq x\left|1-x^{2}\right|+1$ for $x \geq 0$. By the uniform $H^{1}$ bound on $a_{\varepsilon}$, it follows via Sobolev embedding that

$$
\frac{1}{2} E_{c s h}\left(u_{\varepsilon}\right) \leq K|\log \varepsilon|^{2}+C|\log \varepsilon|^{2}+C \varepsilon^{2}|\log \varepsilon|^{4} \leq C|\log \varepsilon|^{2} .
$$

Inequality (13) lets us apply the compactness and lower bound results of Theorem 8 in the Appendix.

We decompose

$$
G_{c s h}\left(u_{\varepsilon}, A_{\varepsilon} ; h_{e x}\right)=G_{c s h}^{1}\left(u_{\varepsilon}, A_{\varepsilon}\right)+G_{c s h}^{2}\left(u_{\varepsilon}, A_{\varepsilon}\right)+G_{c s h}^{3}\left(u_{\varepsilon}, A_{\varepsilon}\right)+G_{c s h}^{4}\left(u_{\varepsilon}, A_{\varepsilon}\right),
$$

where

$$
\begin{aligned}
& G_{c s h}^{1}\left(u_{\varepsilon}, A_{\varepsilon}\right)=E_{c s h}\left(u_{\varepsilon}\right) \\
& G_{c s h}^{2}\left(u_{\varepsilon}, A_{\varepsilon}\right)=\frac{|\log \varepsilon|^{2}}{2} \int_{U}\left|a_{\varepsilon}\right|^{2}+\frac{\mu_{\varepsilon}^{2}|\log \varepsilon|^{2}}{8} \int_{U}\left|\operatorname{curl} a_{\varepsilon}-H\right|^{2} \\
& G_{c s h}^{3}\left(u_{\varepsilon}, A_{\varepsilon}\right)=\frac{|\log \varepsilon|^{2}}{2} \int_{U}\left(\left|u_{\varepsilon}\right|^{2}-1\right)\left|a_{\varepsilon}\right|^{2} \\
& G_{c s h}^{4}\left(u_{\varepsilon}, A_{\varepsilon}\right)=-|\log \varepsilon|^{2} \int_{U} a_{\varepsilon} \cdot v_{\varepsilon} .
\end{aligned}
$$

For $G_{c s h}^{1}$ we use the lower bound of Theorem 8 in the Appendix and obtain

$$
\liminf _{\varepsilon \rightarrow 0} \frac{1}{|\log \varepsilon|^{2}} G_{c s h}^{1}\left(u_{\varepsilon}, A_{\varepsilon}\right) \geq \frac{1}{2}\left(\|v\|_{L^{2}}^{2}+\|\operatorname{curl} v\|_{\mathcal{M}}\right) .
$$

Using the strong $H^{1}$ convergence we estimate

$$
\liminf _{\varepsilon \rightarrow 0} \frac{1}{|\log \varepsilon|^{2}} G_{c s h}^{2}\left(u_{\varepsilon}, A_{\varepsilon}\right) \geq \frac{1}{2}\|a\|_{L^{2}}^{2} .
$$

For the third term, we use Corollary 2.4 of [16], which shows that $\left\|1-\left|u_{\varepsilon}\right|^{2}\right\|_{L^{2}}^{2} \leq$ $C \varepsilon^{2}|\log \varepsilon|^{4}$, hence

$$
\left|G_{c s h}^{3}\left(u_{\varepsilon}, A_{\varepsilon}\right)\right| \leq\left(\int_{U}\left(\left|u_{\varepsilon}\right|^{2}-1\right)^{2}\right)^{1 / 2}\left(\int_{U}\left|A_{\varepsilon}\right|^{4}\right)^{1 / 2} \leq C \varepsilon|\log \varepsilon|^{4} \rightarrow 0 .
$$


The convergence

$$
\frac{1}{|\log \varepsilon|^{2}} G_{c s h}^{4}\left(u_{\varepsilon}, A_{\varepsilon}\right) \rightarrow-\int_{U} a \cdot v
$$

follows from the weak convergence of $v_{\varepsilon}$ in $L^{p}$ with $p<2$ and the strong convergence $a_{\varepsilon} \rightarrow a$ in $L^{q}$ for all $q$ that follows from Rellich-Kondrachov compactness. Summing up the terms, we obtain the lower bound as claimed.

The lower bounds can be complemented by matching upper bounds, yielding $\Gamma$-convergence. The construction is identical to the one used in the $\mu=O(1)$ case, see Sections 6 and 7 of [16].

As an application of the $\Gamma$-liminf energy (10), we compute the asymptotic value of the critical field strength for vortex nucleation. This follows from the solution of a classical obstacle problem, commonly found in free boundary theory. The obstacle problem for Ginzburg-Landau can be found in [20].

Proof of Corollary 4. We compare $G(v, a ; H)$ with $G(0, a ; H)$; the difference is given by

$$
\frac{1}{2} \int_{U}|v|^{2}+\frac{1}{2}\|\operatorname{curl} v\|_{\mathscr{M}}-\int_{U} v \cdot a \geq \frac{1}{2}\|\operatorname{curl} v\|_{\mathscr{M}}-\int_{U} v \cdot a .
$$

Define $z_{\infty}$ as the solution of $\Delta z_{\infty}=-1$ in $U, z_{\infty}=0$ on $\partial U$. Since curl $a=H$ then $H \Delta z_{\infty}=-\operatorname{curl} a$ and $a=-H \operatorname{curl} z_{\infty}$. Integrating by parts, we note that $\int_{U} v \cdot a=H \int_{U} z_{\infty} \operatorname{curl} v$. It follows that

$$
G(v, a ; H)-G(0, a ; H) \geq \int_{U}\left(\frac{1}{2}-\operatorname{sgn}(\operatorname{curl} v) z_{\infty} H\right) d\|\operatorname{curl} v\|,
$$

which is positive for $H \max _{\bar{U}}\left|z_{\infty}\right| \leq \frac{1}{2}$.

Remark 7 . The critical field obtained in the proposition above is identical to the limit as $\mu \rightarrow \infty$ of the critical fields for $\mu=O(1)$ that were calculated in $[16]$.

\section{SMALL $\mu_{\varepsilon}$}

In this section we consider the situation where $\mu_{\varepsilon} \rightarrow 0$ as $\varepsilon \rightarrow 0$. We show that a simple application of the tools of the $\mu=O(1)$ case does not work here by means of a counterexample of a sequence $\left\{u_{\varepsilon}, A_{\varepsilon}\right\}$ where $G_{c s h}\left(u_{\varepsilon}, A_{\varepsilon}\right) \leq$ $K|\log \varepsilon|^{2}$ but $\frac{1}{|\log \varepsilon|} \frac{j\left(u_{\varepsilon}\right)}{\left|u_{\varepsilon}\right|}$ is not bounded in $L^{2}$. In particular we build a lattice of $|\log \varepsilon|$ vortices inside of a box of size $\mu_{\varepsilon}$ with energy of size $|\log \varepsilon|^{2}$. This is possible so long as $\mu_{\varepsilon}$ is not too small. (If $\mu_{\varepsilon}<C \varepsilon|\log \varepsilon|^{\frac{1}{2}}-$ as in the self-dual case - we use a modified construction.) Since $\mu_{\varepsilon} \ll 1$ then the scaled current blows up like $\left\|v_{\varepsilon}\right\|_{L^{2}}^{2} \gtrsim \log \frac{1}{\mu_{\varepsilon}}$. 
Proof of Theorem 5. The idea of the construction is to use $v_{\varepsilon}=\frac{j\left(u_{\varepsilon}\right)}{|\log \varepsilon|}$ and $a_{\varepsilon}=\frac{A_{\varepsilon}}{|\log \varepsilon|}$ and to set $v_{\varepsilon} \approx a_{\varepsilon}$, to let $\frac{\mu_{\varepsilon}^{2}}{4} \int_{U}\left|\operatorname{curl} a_{\varepsilon}\right|^{2} \approx C$ and $\left\|\operatorname{curl} v_{\varepsilon}\right\|_{\mathscr{M}} \approx C$ while $\int_{U}\left|v_{\varepsilon}\right|^{2} \rightarrow \infty$. We do this by letting curl $v_{\varepsilon}$ approach a delta function.

1. To achieve this, we define some auxiliary functions:

$$
\Phi^{s}(r):= \begin{cases}\frac{4}{3} \log 2 s-\frac{1}{3} \log s-\frac{1}{2} & \text { if } r<s \\ \frac{r^{2}}{6 s^{2}}-\frac{1}{3} \log r+\frac{4}{3} \log 2 s-\frac{2}{3} & \text { if } s<r<2 s \\ \log r & \text { else }\end{cases}
$$

Note that $\Phi^{s}(r)$ is a smooth approximation to a solution of $\Delta v=\delta_{0}$ for $s \rightarrow 0$ and that $\int_{B_{\ell}(0)} \Delta \Phi^{s}=2 \pi \int_{s}^{2 s} \frac{2 r}{3 s^{2}} d r=2 \pi$ for $\ell>2 s$. We also set $q_{\varepsilon}(r)=\min \left(1, \frac{r}{\varepsilon}\right)$ Our example will use the domain $U=B_{1}(0)$. We choose $N_{\varepsilon}$ points $a_{i}$ in a square lattice of side length $\frac{\mu_{\varepsilon}}{|\log \varepsilon|^{1 / 2}} \ll \mu_{\varepsilon}$ inside $B_{\mu_{\varepsilon}}(0)$, so $N_{\varepsilon}=\pi|\log \varepsilon|+O\left(|\log \varepsilon|^{1 / 2}\right)$.

We now set $\rho_{\varepsilon}(z)=\prod_{i=1}^{N_{\varepsilon}} q_{\varepsilon}\left(z-a_{i}\right), \Phi_{\varepsilon}(z)=\sum_{i=1}^{N_{\varepsilon}} \Phi^{s}\left(z-a_{i}\right)$, and $\Psi_{\varepsilon}(z)=$ $\sum_{i=1}^{N_{\varepsilon}} \log \left|z-a_{i}\right|$. We further set $V_{\varepsilon}=\operatorname{curl} \Psi_{\varepsilon}$ and $A_{\varepsilon}=\operatorname{curl} \Phi_{\varepsilon}$. To define $u_{\varepsilon}$, we choose a multi-valued function $\phi_{\varepsilon}$ with $\nabla \phi_{\varepsilon}=V_{\varepsilon}$ and set $u_{\varepsilon}=\rho_{\varepsilon} e^{i \phi_{\varepsilon}}$. In our following calculations, we assume $\varepsilon \leq s \leq \frac{\mu_{\varepsilon}}{4|\log \varepsilon|^{1 / 2}}$. We calculate the energy $G_{c s h}\left(u_{\varepsilon}, A_{\varepsilon}\right)$ (the external field is zero in this example). In the case where $\mu_{\varepsilon}<4 \varepsilon|\log \varepsilon|^{1 / 2}$, we will use $\tilde{\mu}_{\varepsilon}=4 \varepsilon|\log \varepsilon|^{1 / 2}$ instead. Note that $\left|\nabla_{A_{\varepsilon}} u_{\varepsilon}\right|^{2}=$ $\left|\nabla \rho_{\varepsilon}\right|^{2}+\rho_{\varepsilon}^{2}\left|V_{\varepsilon}-A_{\varepsilon}\right|^{2}$. From our construction, it follows that

$$
\int_{U}\left|\nabla \rho_{\varepsilon}\right|^{2}+\frac{1}{\varepsilon^{2}} \rho_{\varepsilon}^{2}\left(1-\rho_{\varepsilon}^{2}\right)^{2} \leq C N_{\varepsilon}
$$

For the curl term, we observe that $\operatorname{curl} A_{\varepsilon}=0$ where $\rho_{\varepsilon}=0$, hence

$$
\frac{\mu_{\varepsilon}^{2}}{8} \int_{U} \frac{\operatorname{curl} A_{\varepsilon}^{2}}{\rho_{\varepsilon}^{2}}=N_{\varepsilon} \frac{\mu_{\varepsilon}^{2}}{8} \frac{4}{9 s^{4}} \pi\left(4 s^{2}-s^{2}\right)=\frac{\mu_{\varepsilon}^{2} \pi N_{\varepsilon}}{6 s^{2}} .
$$

For $V_{\varepsilon}-A_{\varepsilon}$, we find that

$$
\left(-\partial_{2}, \partial_{1}\right)\left(\log |z|-\Phi^{s}(z)\right)= \begin{cases}\frac{1}{r^{2}}(-y, x) & r<s \\ \frac{2}{3 r^{2}}(-y, x)+\frac{1}{3 s^{2}}(-y, x) & s<r<2 s \\ 0 & r<2 s\end{cases}
$$


and so since $s$ is chosen such that the fields of different particles $a_{i}$ are disjoint, we can calculate

$$
\begin{aligned}
\int_{U} \rho_{\varepsilon}^{2}\left|V_{\varepsilon}-A_{\varepsilon}\right|^{2} & =2 \pi N_{\varepsilon}\left(\int_{0}^{\varepsilon} \frac{r^{2}}{\varepsilon^{2} r^{2}} r d r+\int_{\varepsilon}^{s} \frac{1}{r^{2}} r d r+\int_{s}^{2 s}\left(\frac{4}{9 r^{2}}+\frac{4}{9 s^{2}}+\frac{r^{2}}{9 s^{4}}\right) r d r\right) \\
& =2 \pi N_{\varepsilon}\left(\frac{1}{2}+\log \frac{s}{\varepsilon}+\frac{4}{9} \log 2+\frac{13}{12}\right) \\
& =N_{\varepsilon}\left(C+\pi \log \frac{s}{\varepsilon}\right) .
\end{aligned}
$$

Summing up, we obtain a total energy of $\left(C+\pi \log \frac{s}{\varepsilon}+C \frac{\mu_{\varepsilon}^{2}}{s^{2}}\right) N_{\varepsilon}$. Choosing $s=\frac{\mu_{\varepsilon}}{4|\log \varepsilon|^{1 / 2}}$, this is bounded by $C|\log \varepsilon|^{2}$ since $\mu_{\varepsilon}<1$.

2. If $\mu_{\varepsilon}<\tilde{\mu}_{\varepsilon}=4 \varepsilon|\log \varepsilon|^{1 / 2}$, the construction has to be done with $\tilde{\mu}_{\varepsilon}$ in place of $\mu_{\varepsilon}$ and $s=\varepsilon$, and the total energy is $\left(C+\pi \log \frac{2 \varepsilon}{\varepsilon}+C \frac{\mu_{\varepsilon}^{2}}{\varepsilon^{2}}\right) N_{\varepsilon}$, and now $\frac{\mu_{\varepsilon}^{2}}{\varepsilon^{2}} \leq 16|\log \varepsilon|$, so the total energy is again bounded by $C|\log \varepsilon|^{2}$.

3. We still need to show the unboundedness of $\frac{1}{|\log \varepsilon|^{2}} \int_{U} \frac{\left|V_{\varepsilon}\right|^{2}}{\left|u_{\varepsilon}\right|^{2}}$. To do so, we estimate it from below by considering only the integral over $B_{1} \backslash B_{2 \mu_{\varepsilon}}$. We note that

$$
\left|V_{\varepsilon}\right|^{2}(z)=\sum_{i=1}^{N_{\varepsilon}} \sum_{j=1}^{N_{\varepsilon}}\left|\frac{z-a_{i}}{\left|z-a_{i}\right|^{2}} \cdot \frac{z-a_{j}}{\left|z-a_{j}\right|^{2}}\right| .
$$

We estimate each of the terms from below, using that $|z| \geq 2 \mu_{\varepsilon}$ and $\left|a_{i}\right|<\mu_{\varepsilon}$. It is clear that $\frac{1}{2}|z| \leq\left|z-a_{i}\right| \leq \frac{3}{2}|z|$.

Hence $\left(z-a_{i}\right) \cdot\left(z-a_{j}\right)=\left|z-a_{i}\right|\left|z-a_{j}\right||\cos \alpha| \geq \frac{1}{4}|z|^{2}|\cos \alpha|$, where $\alpha$ is the angle between $\left(z-a_{i}\right)$ and $\left(z-a_{j}\right)$. Since $\left|a_{i}\right| \leq \mu$ and $|z| \geq 2 \mu$, this angle can be estimated from above, and a short argument in elementary geometry shows that $|\cos \alpha| \geq \frac{\sqrt{3}}{2}$. Hence $\left(z-a_{i}\right) \cdot\left(z-a_{j}\right) \geq \frac{\sqrt{3}}{8}|z|^{2}$, and the contribution of a single term in the sum can be estimated below by $\frac{c}{|z|^{2}}$ (with $c=\frac{2 \sqrt{3}}{81}$ ), and we obtain

$$
\int_{U}\left|V_{\varepsilon}\right|^{2} \geq C N_{\varepsilon}^{2} \int_{2 \mu_{\varepsilon}}^{1} \frac{1}{r} d r=\tilde{C}|\log \varepsilon|^{2} \log \frac{1}{2 \mu_{\varepsilon}},
$$

and this is $\gg|\log \varepsilon|^{2}$ for any $\mu_{\varepsilon} \rightarrow 0$.

4. In the case that $\mu_{\varepsilon}<\tilde{\mu}_{\varepsilon}$, we obtain the same bounds with $\tilde{\mu}_{\varepsilon}$ instead of $\mu_{\varepsilon}$. However, $\log \frac{1}{2 \tilde{\mu}_{\varepsilon}}=\log \frac{1}{8 \varepsilon|\log \varepsilon|^{1 / 2}} \gg 1$ so our unboundedness statement for $\frac{1}{|\log \varepsilon|} V_{\varepsilon}$ still holds.

\section{APPENDIX}

In order to establish Theorem 3 we use the following $\Gamma$-limit result for $E_{c s h} \approx$ $|\log \varepsilon|^{2}$ established in [16]: 
Theorem 8 ([16]). Assume $E_{c s h}\left(u_{\varepsilon}\right) \leq K|\log \varepsilon|^{2}$, for some constant $K$ and $\left\|u_{\varepsilon}\right\|_{L^{\infty}(\partial U)} \geq 1-\frac{1}{|\log \varepsilon|}$.

Set $v_{\varepsilon}=\frac{1}{|\log \varepsilon|} j\left(u_{\varepsilon}\right)$ and $w_{\varepsilon}=\frac{1}{|\log \varepsilon|} J\left(u_{\varepsilon}\right)=\frac{1}{2} \operatorname{curl} v_{\varepsilon}$. Then $\left\{w_{\varepsilon}\right\}$ is precompact in the weak $\left(C^{0, \beta}\right)^{*}$ topology and $\left\{v_{\varepsilon}\right\}$ is bounded in $L^{p}$ for $1 \leq p<2$. Furthermore, if $w_{\varepsilon} \rightarrow w=\frac{1}{2} \operatorname{curl} v$ and $v_{\varepsilon} \rightarrow v$, then also $\frac{v_{\varepsilon}}{\left|u_{\varepsilon}\right|} \rightarrow v$ in $L^{2}$, and the energy satisfies

$$
\liminf _{\varepsilon \rightarrow 0} \frac{1}{|\log \varepsilon|^{2}} E_{c s h}\left(u_{\varepsilon}\right) \geq \frac{1}{2}\left(\|v\|_{L^{2}}^{2}+\|\operatorname{curl} v\|_{\mathcal{M}}\right) .
$$

Conversely, for every $v \in L^{2}\left(U ; \mathbb{R}^{2}\right)$ such that $w=\frac{1}{2} \operatorname{curl} v$ is a Radon measure, there exists a sequence $\left\{u_{\varepsilon}\right\}$ in $H^{1}(U ; \mathbb{C})$ with $\left|u_{\varepsilon}\right|=1$ on $\partial U$ such that $v_{\varepsilon}=\frac{1}{|\log \varepsilon|} j\left(u_{\varepsilon}\right) \rightarrow v$ in $L^{2}$ and $w_{\varepsilon}=\frac{1}{|\log \varepsilon|} J\left(u_{\varepsilon}\right) \rightarrow w$ in $\left(C^{0, \beta}\right)^{*}$ such that the energy satisfies

$$
\lim _{\varepsilon \rightarrow 0} \frac{1}{|\log \varepsilon|^{2}} E_{c s h}\left(u_{\varepsilon}\right)=\frac{1}{2}\left(\|v\|_{L^{2}}^{2}+\|\operatorname{curl} v\|_{\mathcal{M}}\right) .
$$

Proof. Jacobian estimates have been extremely useful in Ginzburg-Landau theory, $[1,3,12,13,14,19]$, and the proof of this theorem follows from the approach of Jerrard-Soner for Ginzburg-Landau, see [12]. In particular the theorem follows quickly from the Jacobian estimate

$$
\left|\int \phi J\left(u_{\varepsilon}\right) d x\right| \leq \pi d\|\phi\|_{L^{\infty}}+C \varepsilon^{\gamma}\|\phi\|_{C^{0,1}}
$$

where $d \approx\left\lfloor\frac{1}{\pi} \int \frac{e_{c s h}\left(u_{\varepsilon}\right)}{|\log \varepsilon|} d x\right\rfloor, \gamma \in(0,1)$, and $C$ depends on $\int \frac{e_{c s h}\left(u_{\varepsilon}\right)}{|\log \varepsilon|} d x$. Estimate (20) follows from Proposition 9 and Proposition 10 below.

We sketch the relationship between the Jacobian

$$
J(u)=\operatorname{det} \nabla u=\frac{1}{2} \operatorname{curl} j(u)
$$

and the energy density $e_{c s h}(u)$ found in (20). Set $\phi \in C_{c}^{0,1}(U)$ a Lipschitz function vanishing on $\partial U$. We define $\Omega(t)=\{x \in U$ such that $\phi(x)>t\}$ then $\partial \Omega(t)$ is a level set $\phi$. Let

$$
\operatorname{Reg}(\phi):=\left\{\begin{array}{c}
t \in\left[0,\|\phi\|_{L^{\infty}}\right] \text { such that } \partial \Omega(t)=\phi^{-1}(t), \\
\partial \Omega(t) \text { rectifiable, and } \mathcal{H}^{1}(\partial \Omega(t))<\infty
\end{array}\right\} .
$$

By the co-area formula $|\operatorname{Reg}(\phi)|=\|\phi\|_{L^{\infty}}$ and $t \in \operatorname{Reg}(\phi)$ implies $\partial \Omega(t)$ is a union of finite Jordan curves, $\Gamma_{i}(t)$. We set, as in [12],

$$
\Gamma(t):=\cup\left\{\text { components of } \partial \Omega(t) \text { such that } \min _{x \in \Gamma_{i}(t)}|u|>\frac{1}{2}\right\} .
$$

We set $d \in \mathbb{Z}^{+}$and define

$$
D_{d}:=\{t \in \operatorname{Reg}(\phi): \Gamma(t) \text { is nonempty and }|\operatorname{deg}(u ; \Gamma(t))| \geq d+1\} .
$$


We will choose $d$ to be the least integer multiple of $\pi|\log \varepsilon|$ of the energy. Let us define

$$
E_{\phi}(u)=\int_{\operatorname{spt}(\phi)} e_{c s h}(u) d x
$$

for short. The first estimate is rather direct and follows in spirit of [12]:

Proposition 9. Suppose $U \subset \mathbb{R}^{2}$ and $u \in H^{1}(U ; \mathbb{C})$ then

$$
\begin{aligned}
\left|\int_{U} \phi J(u) d x\right| \leq & \left(\pi d+\varepsilon^{\frac{1}{2}}\right)\|\phi\|_{L^{\infty}} \\
& +\varepsilon^{\frac{1}{3}}\|\nabla \phi\|_{L^{\infty}}\left[2 E_{\phi}^{2}(u)+3 E_{\phi}(u)+\frac{|\operatorname{spt}(\phi)|}{4}\right] \\
& +\frac{\left|D_{d}\right|}{4} E_{\phi}(u)
\end{aligned}
$$

for any $\varepsilon \leq 1$.

In order to use (21) and close the estimate we need to control $\left|D_{d}\right|$. This is estimated by the following result.

Proposition 10. Suppose $|u| \geq 1-\frac{1}{|\log \varepsilon|}$ on $\partial U$ and let $\varepsilon \in\left(0, e^{-2}\right)$. Define

$$
\varepsilon^{\alpha_{\varepsilon}}=\varepsilon|\log \varepsilon|^{2} \int_{U} e_{c s h}(u) d x \quad \eta_{\varepsilon}=\frac{1}{1-\frac{2}{|\log \varepsilon|}}>1
$$

then

$$
\left|D_{d}\right| \leq 8 \varepsilon^{\alpha_{\varepsilon}}\|\nabla \phi\|_{L^{\infty}}\left(1+\exp \left(\frac{\eta_{\varepsilon}}{\pi d_{\star}} \int_{V} e_{c s h}(u) d x\right)\right)
$$

where $d_{\star}=d+1$. Here $V \equiv \dot{\cup}_{j} B_{r_{j}}$ is the union of disjoint balls $B_{r_{j}}$ with $B_{r_{j}} \subseteq \operatorname{spt}(\phi) \subseteq U$ and $\sum_{j} r_{j}=\frac{\left|D_{d}\right|}{2\|\nabla \phi\|_{L^{\infty}}}$.

Proposition 10 provides a good estimate for $\left|D_{d}\right|$ when $d \approx\left\lfloor\int_{V} \frac{e_{c s h}\left(u_{\varepsilon}\right)}{\pi|\log \varepsilon|}\right\rfloor$, and this estimate follows from a variation of the Jerrard [11] and Sandier [18] vortex ball method. There is also a different approach [21] that can be taken to the estimate of $\left|D_{d}\right|$.

\section{REFERENCES}

[1] Alberti, G., Baldo, S., and Orlandi, G. Variational convergence for functionals of Ginzburg-Landau type. Indiana Univ. Math. J. 54 (2005), no. 5, 1411-1472.

[2] Bethuel, F., Brezis, H. and Helein, F. Ginzburg-Landau Vortices Birkhäuser, Boston, (1994).

[3] Bethuel, F., Brezis, H., and Orlandi, G. Asymptotics for the Ginzburg-Landau equation in arbitrary dimensions. J. Funct. Anal. 186 (2001), 432-520. 
[4] Caffarelli, L. and Yang, Y. Vortex condensation in the Chern-Simons-Higgs model: An existence theorem Comm. Math. Phys. 168 (1995), 321-336.

[5] Chae D. and Kim, N. Topological multivortex solutions of the self-dual Maxwell-ChernSimons-Higgs system J. Differential Equations 134 (1997), 154-182.

[6] Han, J. and Jang, J. Self-dual Chern-Simons vortices on bounded domains, Lett. Math. Phys. 64 (2003), 45-56.

[7] Han, J. and Kim, N. Nonself-dual Chern-Simons and Maxwell-Chern-Simons vortices on bounded domains. J. Funct. Anal. 221 (2005), 167-204.

[8] Hong, J., Kim, Y., and Pac, P.Y. Multivortex solutions of the Abelian Chern-Simons vortices Phys. Rev. Lett. 64 (1990), 2230-2233.

[9] Jackiw, R. and Weinberg, W. Self-dual Chern-Simons vortices Phys. Rev. Lett. 64 (1990), 2234-2237.

[10] Jaffe, A. and Taubes, C. Vortices and monopoles. Structure of static gauge theories, Progress in Physics, 2. Birkhäuser, Boston, Mass., (1980).

[11] Jerrard, R. L. Lower bounds for generalized Ginzburg-Landau functionals, SIAM J. Math. Anal. 30 (1999), 721-746.

[12] Jerrard, R. L. and Soner, H. M. The Jacobian and the Ginzburg-Landau energy. Calc. Var. Partial Differential Equations 14 (2002), 151-191.

[13] Jerrard, R. L. and Soner, H. M. Limiting behavior of the Ginzburg-Landau functional. J. Funct. Anal. 192 (2002), 524-561.

[14] Jerrard, R. L. and Spirn, D. Refined Jacobian estimates and the Ginzburg-Landau energy, submitted to Indiana U Math. J.

[15] Jerrard, R. L. and Spirn, D. Refined Jacobian estimates and the Gross-Pitaevsky equations, submitted to Arch. Rat. Mech. Anal.

[16] Kurzke, M. and Spirn, D. Gamma limit of the nonself-dual Chern-Simons-Higgs energy, submitted to Ann. Inst. H. Poincare.

[17] Ricciardi, T. and Tarantello, G. Vortices in the Maxwell-Chern-Simons theory. Comm. Pure Appl. Math. 53 (2000), 811-851.

[18] Sandier, E. Lower bounds for the energy of unit vector fields and applications J. Funct. Anal. 152 (1998), 379-403.

[19] Sandier, E. and Serfaty, S. A product-estimate for Ginzburg-Landau and corollaries. J. Funct. Anal. 211 (2004), 219-244. 
[20] Sandier, E. and Serfaty, S. A rigorous derivation of a free-boundary problem arising in superconductivity. Ann. Sci. École Norm. Sup. (4) 33 (2000), 561-592.

[21] Sandier, E. and Serfaty, S. Vortices in the Magnetic Ginzburg-Landau Model, Birkhäuser, Progress in Nonlinear PDEs. preprint.

[22] Serfaty, S. Local minimizers for the Ginzburg-Landau energy near critical magnetic field. I. Commun. Contemp. Math. 1 (1999), 213-254.

[23] Tarantello, G. Multiple condensate solutions for the Chern-Simons-Higgs theory, J. Math. Phys. 37 (1996), 3769-3796.

[24] Yang, Y. Solitons in field theory and nonlinear analysis. Springer Monographs in Mathematics. Springer-Verlag, New York, 2001.

Institut für Mathematik, Humboldt-Universität Zu Berlin, Unter den Linden 6, 10099 Berlin, Germany

E-mail address: kurzkem@mathematik.hu-berlin.de

School of Mathematics, University of Minnesota, Minneapolis, MN 55455

E-mail address: spirn@math.umn.edu 